\title{
Extracellular fluid adjusted for body size is contracted in hypertension
}

\author{
Hong Seog Seo, Eung Ju Kim, Sun Won Kim, Sung Il Im, Jin Oh Na, Cheol Ung Choi, Hong Euy Lim, \\ Jin Won Kim, Seung-Woon Rha and Chang Gyu Park
}

Extracellular fluid (ECF) is associated with blood pressure, but reports on the status of the ECF volume in hypertension have been inconsistent. The aim of this study was to assess the ECF volume status in hypertensives with regard to body size in a large cohort. We performed a single-center case-control observation study for patients who visited the outpatient hypertension clinic and health examination center. For all eligible participants, we examined the body composition, including fluid compartments, using a noninvasive bioimpedance analysis. Of 2934 subjects (women $1365,57.5 \pm 12.2$ years), 1166 subjects were normotensive and 1768 subjects were hypertensive. The ECF volume was larger in female hypertensives than in normotensives but was not different between the male groups. However, the relative ECF, defined as the ratio of ECF to body mass index $(\mathrm{BMI})$, was significantly lower in hypertensives of both genders $(\boldsymbol{P}<0.001)$. ECF revealed an almost twofold stronger correlation with the fat-free mass (FFM) ( $r=0.9$ in both genders) than with the fat mass, BMI or waist circumference and a negative correlation with age. In contrast, the relative ECF was positively correlated only with the FFM and inversely correlated with the other factors. In the multivariate logistic regression analysis, an increase of 1 s.d. in the relative ECF decreased the relative risk of hypertension by $30 \%$ in women (odds ratio (OR), $0.70 ; 95 \%$ confidence interval $(\mathrm{CI}), 0.56-0.87)$ and by $28 \%$ in men (OR, $0.72 ; 95 \% \mathrm{Cl}, 0.60-0.86)$, but the ECF was not independently associated with hypertension in either gender. The ECF with regard to the body size was contracted in hypertensives and independently associated with hypertension, whereas the absolute ECF volume was not.

Hypertension Research (2013) 36, 916-921; doi:10.1038/hr.2013.68; published online 11 July 2013

Keywords: body composition; body mass index; extracellular fluid; hypertension

\section{INTRODUCTION}

Hypertension affected more than a quarter of the world adult population in 2000 , and its prevalence is growing, as it is predicted to increase up to $29 \%$ - to 1.56 billion people—by $2025 .^{1}$ Therefore, the global initiative is increasingly stressed in favor of the more precise management of hypertension. However, $95 \%$ of hypertensive subjects are classified as having primary hypertension, indicating that there is no clearly known origin that might explain the initiation of hypertension.

In general, the elevation of extracellular fluid (ECF) increases the preload, which eventually contributes to the generation of hypertension. ${ }^{2}$ However, the volume of ECF in hypertension has been reported to be diverse (expanded, normal or reduced), and most results were from small-sized studies. ${ }^{3-10}$ Moreover, those studies mostly evaluated only the absolute ECF amount, although ECF is largely dependent on the size and composition of the body. ${ }^{11}$ As the hydration of fat mass (FM) differs markedly from that of the fat-free mass (FFM), the recognition of these differences would be important for a better understanding of the relationship of ECF to hypertension.
Recently, bioelectrical impedance analysis (BIA) has become increasingly popular for estimating body composition, including extracellular and intracellular fluids, FM and FFM. This method is advantageous because it noninvasively and inexpensively assesses the aforementioned properties in a wide range of subjects, regardless of age and body shape. The validity and reproducibility of BIA for estimating body composition is supported by numerous publications in the literature with and without accompanying reference methods. ${ }^{12-25}$ The aim of this study was to assess the ECF volume using BIA in hypertensives with regard to body size in a large cohort.

\section{METHODS}

Study subjects

We recruited Korean subjects, aged 18-90 years, who visited the Cardiovascular Center Outpatient Hypertension Clinic and Health Examination Center of Korea University Guro Hospital, Seoul, Korea from May 2007 to April 2009. Of 4143 subjects, 342 subjects were excluded owing to incomplete data, and 867 subjects were excluded according to the following exclusion criteria: (1) age $<18$ years; (2) existence of secondary hypertension; (3) existence of organ 
failure (kidney, heart and liver) or other major disease; (4) clinically detectable fluid retention (peripheral edema, neck vein distension or lymphedema); (5) use of drugs such as diuretics, glucocorticoids, thiazolidinedione, anorexigens, antidepressants and neuroleptics; and (6) pregnancy. Finally, the 2934 eligible subjects for this study included 1768 patients with primary hypertension and 1166 normotensive subjects. All of the subjects were provided written informed consent, and the local Institutional Review Board, in accordance with the Declaration of Helsinki of the World Medical Association, approved the study protocol.

\section{Clinical measurements}

The medical history and physical characteristics were obtained by the physicians, and trained research nurses measured the anthropometric characteristics. After at least $10 \mathrm{~min}$ of rest, the sitting systolic and diastolic blood pressure (BP) (first and fifth Korotkoff sounds) were recorded twice on a 2-min basis at the office by the research nurse using a random zero mercury sphygmomanometer fitted with an appropriate cuff size. The BP records were averaged for use in the analysis. All blood samples were obtained in the morning in a fasting state. The following clinical and demographic parameters were recorded as follows: age, sex, hypertension (hypertension treated with antihypertensive medications or two or more BP recordings at the office $>140 / 90 \mathrm{~mm} \mathrm{Hg}$ ), diabetes mellitus (diabetes treated with diet, medications or both or a fasting serum glucose of $>126 \mathrm{mg} \mathrm{dl}^{-1}$ ) and dyslipidemia (treated hypercholesterolemia or a fasting serum total cholesterol $\geqslant 240 \mathrm{mg} \mathrm{dl}^{-1}$, triglycerides $\geqslant 200 \mathrm{mg} \mathrm{dl}^{-1}$, high-density lipoprotein cholesterol $<40 \mathrm{mg} \mathrm{dl}^{-1}$ or low-density lipoprotein cholesterol $\geqslant 160 \mathrm{mg} \mathrm{dl}^{-1}$ ). With regard to smoking status, current smokers were defined as actively smoking within the past 12 months. The mean frequency of alcohol consumption was collapsed into a dichotomous variable, $<1$ or $\geqslant 1$ per week. The body mass index (BMI) was calculated as the weight in kilograms divided by the square of the height in meters. Obesity was defined as a BMI of at least $25 \mathrm{~kg} \mathrm{~m}^{-2}$ using the World Health Organization Asia-Pacific guidelines. ${ }^{26}$

\section{BIA measurements}

The body composition and volume of the fluid compartments were obtained by segmental bioelectrical impedance using eight tactile electrodes, according to the manufacturer's instructions (InBody 3.0; Biospace, Seoul, Korea). The subjects were examined in the morning after overnight fasting $(\geqslant 8 \mathrm{~h})$ and emptying their bladder at room temperature. After at least $5 \mathrm{~min}$ of rest, the participants wiped the bottom of their feet with a proprietary electrolyte tissue before standing on the electrodes embedded in the scale platform and stood on the platform with electrodes attached to their hands and feet. Thus, a pair of electrodes was attached to the surface of each hand and foot at the thumb, palm, fingers, ball of the foot and heel. These electrodes were connected to current and voltage terminals from an impedance meter via electronic on-off switches, which were regulated by a microprocessor. The participants were instructed to stand upright, grasp the handles of the analyzer, abduct his or her arms and then remain still during the assessment. The frequencies used by the InBody 3.0 analyzer were 5, 50, 250 and $500 \mathrm{kHz}$. The segmental impedance of the right arm, left arm, trunk, right leg and left leg were measured at each frequency. Low-level frequencies (for example, $1-50 \mathrm{kHz}$ ) rely on the conductive properties of the ECF, whereas at the high-level frequencies (for example, $250 \mathrm{kHz}$ ), the conductive properties of both intracellular and extracellular water are instrumental. FFM and FM can be calculated from multiple frequency bioimpedance analysis following the application of previously determined constants that represent the contribution of water to FFM. The chemical composition of FFM is assumed to be relatively constant, with a density of $1.1 \mathrm{~kg} \mathrm{~m}^{-3}$ at $37^{\circ} \mathrm{C}$ and a water content of $73 \%{ }^{27}$ Thus, the FFM was calculated by multiplying the values for the water volumes of each segment by 1.37 .

\section{Statistical analysis}

All continuous variables used were normally distributed and expressed as the mean \pm s.d. Differences between groups were examined using Student's $t$-test. Discrete variables were presented as the total number (percentage) and compared using the $\chi^{2}$-test. Pearson's correlation coefficients were calculated to evaluate the relationships among the numeric study variables. In addition, the ratio of ECF to BMI was categorized into quartiles, and intergroup differences were tested using an analysis of variance or the $\chi^{2}$-test if appropriately applicable. The associations between ECF or the ratio of ECF to BMI and hypertension were assessed after the standardization of ECF and the ratio of ECF to BMI to a mean of 0 and a s.d. of 1 using multivariate logistic regression analysis. All statistical results were based on two-sided tests, and a $P$-value $<0.05$ was considered statistically significant. The data were analyzed using SPSS (Statistical Package for the Social Sciences) for Windows (version 12.0; SPSS, Chicago, IL, USA).

\section{RESULTS}

The clinical and anthropometric characteristics of the subjects are shown in Table 1 . The hypertensive subjects appeared to be older in age and to have higher waist circumference, BMI and FM in both genders. They were also more concerned about a higher frequency of obesity, diabetes, dyslipidemia and medication use. No significant differences in the prevalence of current smokers, frequency of alcohol consumption, levels of hematocrit, intracellular fluid, ECF and FFM were found in the comparisons between the groups in men. However, the prevalence of current smokers and the levels of hematocrit, ECF and FFM were higher in the female hypertensives than in the normotensives. The ratio of ECF to BMI was significantly lower in the hypertensives than in the normotensives for both women $\left(38.1 \pm 4.9\right.$ vs. $\left.39.8 \pm 5.4\left(\times 10^{-2} 1 /\left(\mathrm{kg} \mathrm{m}^{-2}\right)\right), P<0.001\right)$ and $m e n$ $\left(50.8 \pm 6.0\right.$ vs. $\left.52.5 \pm 6.2\left(\times 10^{-2} \mathrm{l} /\left(\mathrm{kg} \mathrm{m}^{-2}\right)\right), P<0.001\right)$.

Table 2 displays Pearson's correlation analysis according to gender, representing the relationships among ECF, BMI, the ratio of ECF to BMI and the study variables. The ECF measurements were significantly associated with FM, FFM, BMI and waist circumference $(P<0.001$ for all $)$ positively and with age negatively $(P<0.001)$ in both genders. In particular, because the correlation coefficient for FFM was $\sim 0.9$, it was nearly twice as strongly correlated with FFM compared with FM, BMI or waist circumference. The BMI was more strongly correlated with FM and waist circumference than with FFM. The ratio of ECF to BMI showed a significant negative correlation with age, FM, BMI and waist circumference and a significant positive correlation with FFM in both genders.

The cohort was gender specifically divided into quartiles on the basis of the ratio of ECF to BMI. As the ratio of ECF to BMI decreased, there were increases in age and $\mathrm{BP}$ and higher prevalences of obesity, hypertension, diabetes and dyslipidemia (Table 3).

In an age-adjusted model, hypertension was positively associated with ECF and was significantly inversely associated with the ratio of ECF to BMI in both genders (Table 4). However, the association of ECF did not remain significant after further adjustment for BMI and other variables, whereas the association of the ratio of ECF to BMI remained significant after the multivariable adjustment. As the ratio of ECF to BMI increased by 1 s.d., the odds ratio (OR) of hypertension was 0.70 (95\% confidence interval (CI), 0.56-0.87; $P<0.001)$ in women and 0.72 (95\% CI, $0.60-0.86 ; P<0.001)$ in men.

Concerning the 2188 subjects who were untreated with antihypertensive agents, there was a significant inverse correlation of the ratio of ECF to BMI with BP ( $r=-0.132$ for systolic BP, $r=-0.125$ for diastolic BP in women and $r=-0.142$ for systolic BP, $r=-0.197$ for diastolic BP in men, $P<0.001$ for all)

\section{DISCUSSION}

The major findings in the present study enable us to identify the importance of the relative ECF, defined as the ratio of ECF to $\mathrm{BMI}$, in hypertension. The relative ECF significantly decreased in 
Table 1 Clinical and anthropometric characteristics of the subjects

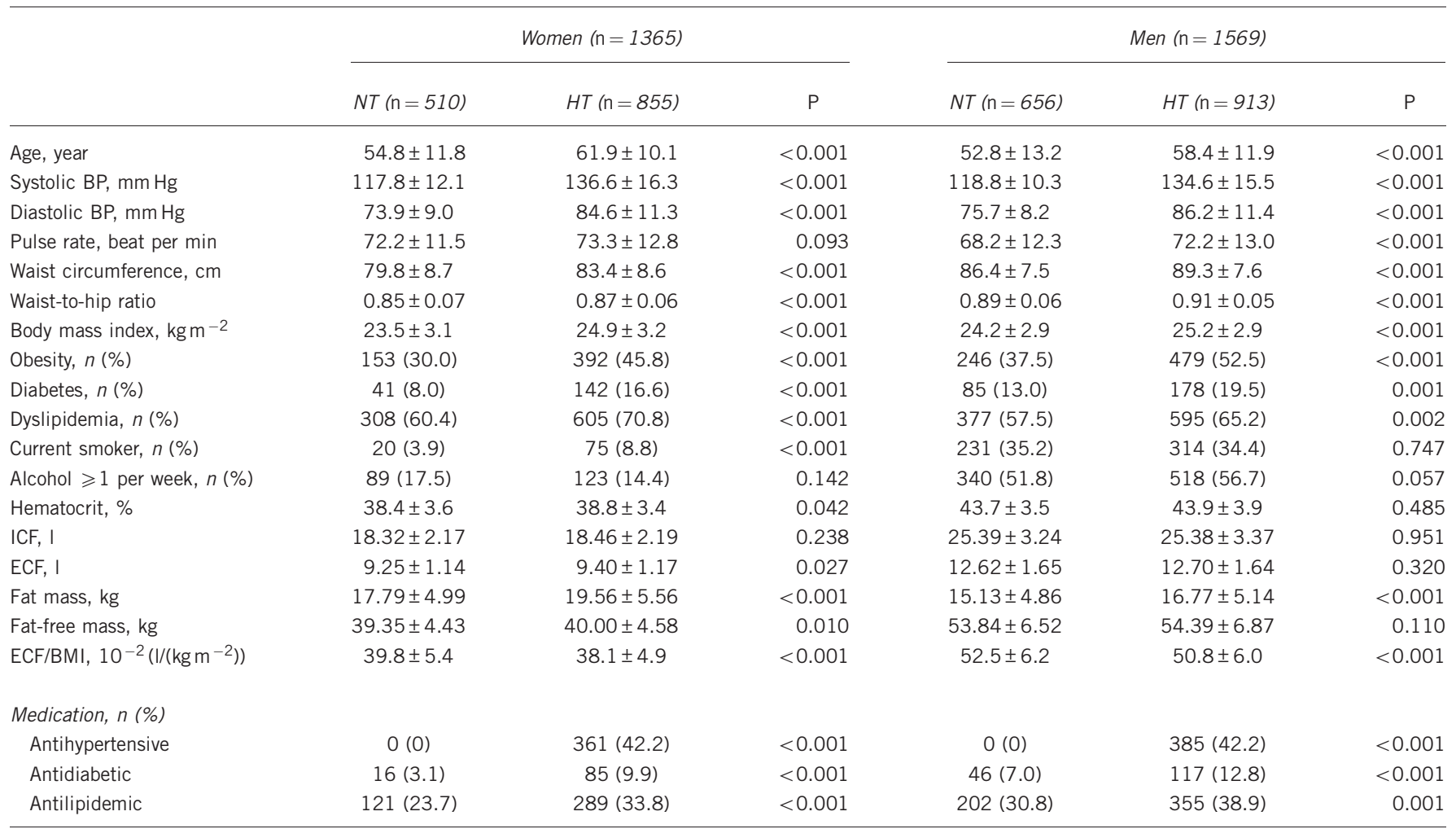

Abbreviations: BMI, body mass index; BP, blood pressure; ECF, extracellular fluid; ICF, intracellular fluid; HT, hypertensives; NT, normotensives.

Table 2 Correlation analysis between ECF, BMI, the ratio of ECF to BMI and study variables

\begin{tabular}{|c|c|c|c|c|c|c|c|c|c|c|c|c|}
\hline & \multicolumn{6}{|c|}{ Women } & \multicolumn{6}{|c|}{ Men } \\
\hline & \multicolumn{2}{|c|}{ ECF } & \multicolumn{2}{|c|}{$B M I$} & \multicolumn{2}{|c|}{$E C F / B M I$} & \multicolumn{2}{|c|}{ ECF } & \multicolumn{2}{|c|}{$B M I$} & \multicolumn{2}{|c|}{$E C F / B M I$} \\
\hline & $r$ & $\mathrm{P}$ & r & $\mathrm{P}$ & r & $\mathrm{P}$ & r & $\mathrm{P}$ & $r$ & $P$ & $r$ & $P$ \\
\hline Age & -0.115 & $<0.001$ & 0.157 & $<0.001$ & -0.271 & $<0.001$ & -0.284 & $<0.001$ & -0.132 & $<0.001$ & -0.188 & $<0.001$ \\
\hline Fat mass & 0.442 & $<0.001$ & 0.931 & $<0.001$ & -0.518 & $<0.001$ & 0.414 & $<0.001$ & 0.877 & $<0.001$ & -0.412 & $<0.001$ \\
\hline Fat-free mass & 0.896 & $<0.001$ & 0.488 & $<0.001$ & 0.330 & $<0.001$ & 0.915 & $<0.001$ & 0.611 & $<0.001$ & 0.375 & $<0.001$ \\
\hline BMI & 0.467 & $<0.001$ & 1 & - & -0.565 & $<0.001$ & 0.535 & $<0.001$ & 1 & - & -0.408 & $<0.001$ \\
\hline Waist circumference & 0.446 & $<0.001$ & 0.786 & $<0.001$ & -0.407 & $<0.001$ & 0.544 & $<0.001$ & 0.796 & $<0.001$ & -0.224 & $<0.001$ \\
\hline
\end{tabular}

Abbreviations: BMI, body mass index; ECF, extracellular fluid.

hypertensives compared with that in normotensives and showed an independent inverse relationship with hypertension in the multivariate analysis.

Although ECF itself was also significantly associated with hypertension in an age-adjusted model, the association was no longer significant after further adjustment for BMI. This finding suggested that the association of ECF with hypertension was largely affected by BMI, which had a moderate correlation with ECF and is one of the strongest risk factors for hypertension. Therefore, ECF per se seemed not to be independently associated with hypertension in this study.

The absolute value of ECF is not legitimately predictive of the physiological standard because it is influenced by body size and composition. ${ }^{11}$ However, ECF expansion could elevate the hydrostatic pressure in the artery and hence increase BP. This relationship could hold, at least in the short-term, such as in large-volume intravenous fluid resuscitation in a shock state. The relationship between ECF expansion and hypertension is also widely believed to depend on the development of hypervolemia, increased cardiac output and the subsequent rise in the total peripheral resistance reducing volume expansion and normalizing systemic flow while maintaining a high BP. ${ }^{6}$ Although this sequence of events has been demonstrated in some human and experimental forms of hypertension, but not in all, it is considered as one of the mechanisms of obesity-induced hypertension. $^{28}$

If the body weight gain is mainly from FM (14\% water content per unit weight), ${ }^{29}$ there should be a smaller ECF expansion compared with that from FFM (70-80\% water content per unit weight). ${ }^{30}$ However, the BMI increase was predominantly related to FM rather 
Table 3 Variables stratified by quartiles of the ratio of ECF to BMI

\begin{tabular}{|c|c|c|c|c|c|c|c|c|c|c|}
\hline & \multicolumn{5}{|c|}{ Women $(n=1365)$} & \multicolumn{5}{|c|}{$\operatorname{Men}(n=1569)$} \\
\hline & $\begin{array}{c}\text { First } \\
\text { quartile } \\
(\mathrm{n}=341)\end{array}$ & $\begin{array}{l}\text { Second } \\
\text { quartile } \\
(\mathrm{n}=342)\end{array}$ & $\begin{array}{c}\text { Third } \\
\text { quartile } \\
(\mathrm{n}=341)\end{array}$ & $\begin{array}{c}\text { Fourth } \\
\text { quartile } \\
(\mathrm{n}=341)\end{array}$ & $P$ & $\begin{array}{c}\text { First } \\
\text { quartile } \\
(\mathrm{n}=392)\end{array}$ & $\begin{array}{l}\text { Second } \\
\text { quartile } \\
(\mathrm{n}=392)\end{array}$ & $\begin{array}{c}\text { Third } \\
\text { quartile } \\
(\mathrm{n}=393)\end{array}$ & $\begin{array}{c}\text { Fourth } \\
\text { quartile } \\
(\mathrm{n}=392)\end{array}$ & $P$ \\
\hline ECF/BMI, $10^{-2}\left(\mathrm{I} /\left(\mathrm{kg} \mathrm{m}^{-2}\right)\right)$ & $32.6 \pm 2.2$ & $36.9 \pm 0.9$ & $40.1 \pm 1.0$ & $45.4 \pm 3.3$ & & $44.0 \pm 3.0$ & $49.3 \pm 1.1$ & $53.2 \pm 1.2$ & $59.4 \pm 3.5$ & \\
\hline Diastolic BP, mm Hg & $82.3 \pm 11.3$ & $80.7 \pm 10.9$ & $80.6 \pm 11.9$ & $79.0 \pm 12.4$ & $<0.001$ & $83.9 \pm 10.8$ & $82.7 \pm 11.1$ & $81.7 \pm 11.7$ & $79.1 \pm 11.5$ & $<0.001$ \\
\hline Obesity, $n(\%)$ & $244(71.6)$ & $149(43.6)$ & $100(29.3)$ & $52(15.2)$ & $<0.001$ & $264(67.3)$ & $205(52.3)$ & 157 (39.9) & $99(25.3)$ & $<0.001$ \\
\hline Hypertension, $n(\%)$ & $245(71.8)$ & $228(66.7)$ & $194(56.9)$ & $188(55.1)$ & $<0.001$ & $260(66.3)$ & $244(62.2)$ & $222(56.5)$ & $187(47.7)$ & $<0.001$ \\
\hline Diabetes, $n(\%)$ & $59(17.3)$ & $47(13.7)$ & $34(10.0)$ & $43(12.6)$ & 0.043 & $72(18.4)$ & $69(17.6)$ & $61(15.5)$ & $61(15.6)$ & 0.624 \\
\hline Dyslipidemia, $n(\%)$ & $261(76.5)$ & $237(69.3)$ & $236(69.2)$ & $179(52.5)$ & $<0.001$ & $270(68.9)$ & $268(68.4)$ & $235(59.8)$ & $199(50.8)$ & $<0.001$ \\
\hline ECF, I & $8.7 \pm 0.9$ & $9.2 \pm 1.0$ & $9.5 \pm 1.0$ & $10.0 \pm 1.3$ & $<0.001$ & $11.6 \pm 1.4$ & $12.4 \pm 1.3$ & $12.9 \pm 1.4$ & $13.8 \pm 1.7$ & $<0.001$ \\
\hline
\end{tabular}

Abbreviations: BMI, body mass index; BP, blood pressure; ECF, extracellular fluid.

$P$-values represent overall differences across groups as determined by analysis of Variance for continuous variables and Pearson's $\chi^{2}$-test for categorical variables.

Table 4 Association of ECF and ECF/BMI with hypertension per s.d. of them ${ }^{a}$

\begin{tabular}{|c|c|c|c|c|}
\hline & \multicolumn{2}{|c|}{ Women $(n=1365)$} & \multicolumn{2}{|c|}{ Men $(n=1569)$} \\
\hline & Odds ratio $(95 \% \mathrm{Cl})$ & $\mathrm{P}$ & Odds ratio $(95 \% \mathrm{Cl})$ & $\mathrm{P}$ \\
\hline Age adjustment & $1.51(1.20-1.89)$ & $<0.001$ & $1.31(1.13-1.51)$ & $<0.001$ \\
\hline Age and BMI adjustment & $1.08(0.83-1.40)$ & 0.568 & $0.94(0.79-1.12)$ & 0.495 \\
\hline Age, BMI and multivariable adjustment ${ }^{\mathrm{b}}$ & $1.05(0.78-1.40)$ & 0.758 & $0.97(0.79-1.19)$ & 0.740 \\
\hline \multicolumn{5}{|l|}{ ECF/BMI } \\
\hline Age adjustment & $0.73(0.60-0.89)$ & 0.002 & $0.73(0.63-0.85)$ & $<0.001$ \\
\hline Age and multivariable adjustment ${ }^{\mathrm{b}}$ & $0.70(0.56-0.87)$ & 0.002 & $0.72(0.60-0.86)$ & $<0.001$ \\
\hline
\end{tabular}

Abbreviations: BMI, body mass index; ECF, extracellular fluid.

${ }^{a}$ ECF and ECF/BMI have been standardized to a mean of 0 and s.d. of 1 to facilitate comparison.

bincludes pulse rate, diabetes, dyslipidemia, smoking, hypertension treatment, diabetes treatment and lipid-lowering therapy. But smoking is only used for women and pulse rate is only used for men.

than to FFM expansion during adulthood, similar to our result (Table 2). Hence, the relative ECF adjusted for body size inevitably declines as the BMI increases, in contrast to the absolute ECF volume.

In the healthy average $70 \mathrm{~kg}$ adult human, the total body water is $\sim 60 \%$ of the body weight or $\sim 421 .{ }^{11}$ This percentage can change, depending on age, gender and degree of adiposity. As a person grows older or becomes fatter, the percentage of total body weight, that is, fluid gradually decreases. ${ }^{11}$ We could hypothesize that a decrease in the relative ECF compared with the physiological fluid balance might lead to the compensatory activation of the renin-angiotensinaldosterone system (RAAS), thereby contributing, at least in part, to the development of hypertension. This effect could be one of the reasons why obese people have shown a higher prevalence of hypertension in the general population ${ }^{2}$ and might account for the phenomena found in obesity-induced hypertension, such as impaired natriuresis and enhanced circulating RAAS activation despite the remarkable absolute volume expansion and sodium retention. ${ }^{28}$

Moreover, renin, angiotensin II, angiotensinogen and angiotensinogen II receptors are found in abundance in adipose mass, suggesting that a local tissue RAAS occurs at the adipocyte level, ${ }^{31}$ possibly in response to the absence of fluid around this region. In the present study, as the level of the relative ECF decreased, there were higher proportions of obesity and hypertension (Table 3). Supporting data indicating that a decrease in the BP was closely related to a reduction in the abdominal $\mathrm{FM}^{32}$ and that weight reduction caused the elevation of ECF have been reported. ${ }^{4,33}$ Even a modest reduction in weight can lead to a meaningful reduction in RAAS activity in plasma and adipose tissue. ${ }^{34}$

If the RAAS was involved in the relationship between the relative ECF and hypertension, the relationship could be affected by RAAS blockers. However, a further adjustment of the multiple logistic regression analysis with respect to the use of RAAS blockers did not significantly change the results in Table 4 (data not shown). This study was not prospectively designed to reveal the mechanism responsible for the relationship between the relative ECF and hypertension, and the dose and type of RAAS blockers used in the study varied. In addition, the RAAS blocker dose required to lower the BP might not be sufficient to affect the local RAAS. 
Although the relative ECF can reflect both body size and composition, including adiposity, and thus enable the critical characterization of the water balance in the body; few studies investigating the association of the relative ECF with primary hypertension exist. Raison et al. ${ }^{4}$ reported that the ECF volume increased in both obese and non-obese hypertensives when expressed in milliliters or milliliters per unit of height but decreased when expressed in milliliters per kilogram in obese hypertensives $(n=16)$ but not in non-obese hypertensives $(n=16)$ compared with control subjects $(n=18)$. Tarazi et al. ${ }^{35}$ also reported similar results. Compared with normotensives, the plasma volume was significantly contracted when expressed per unit height or weight in hypertensive men with a diastolic pressure over $105 \mathrm{~mm} \mathrm{Hg}$ in their study. However, the previous studies included only a small number of subjects, and ECF was separately divided by the body height or weight; therefore, it could not provide definite insight into the association of the relative ECF with hypertension.

The present study also has several aspects to be discussed. First, due to the nature of a cross-sectional study, we are limited to inferring the absolute causal relationship. Whether these body fluid changes represent the cause or effect of the BP rise is not known. Second, many hypertensive patients were treated with antihypertensive agents. Although we excluded the patients treated with diuretics and other potential agents, that can influence the volume status, the ECF volume might be affected by other drugs. Third, the gold standard for the measurement of water spaces is the isotopic dilution of labeled water or bromide. ${ }^{13,22}$ However, these procedures are invasive because they require blood samples and are expensive due to dosage by mass spectrometry. Therefore, the implementation of these measurements for a large number of subjects is very difficult. These factors also explain why there have only been small-sized clinical studies with potential bias or experimental animal studies and may account for the many conflicting results about the volume status in uncomplicated hypertension. However, we could perform a larger human study using BIA, which is noninvasive, easy, inexpensive, validated and reproducible, for the estimation of body fluid compartments. ${ }^{12-25}$

This is the largest-scale human study so far evaluating the relationship between hypertension and fluid compartments. In particular, we adopted the concept of relative ECF using BMI as a reference index. The relative ECF volume with regard to body size was reduced in hypertensive patients and independently inversely associated with hypertension, whereas the absolute ECF volume was not Although this study did not reveal the causal relationship between ECF or relative ECF and hypertension, these findings could provide new insights into understanding the pathophysiology of primary hypertension.

\section{CONFLICT OF INTEREST}

The authors declare no conflict of interest.

\section{ACKNOWLEDGEMENTS}

This research project would not have been possible without the support of many people. We thank Prof Dr Dong Joo Oh, who was abundantly helpful and offered his invaluable assistance, support and guidance. We also thank members of the supervisory committee, Dr Seong Hwan Kim, Dr Seong Woo Han, Seon Mee Kim and Dr Youn Seon Choi, and this study would not have been successful without their knowledge and assistance. This study was supported by a grant from the Korean Health Technology R\&D Project, Ministry for Health, Welfare \& Family Affairs, Republic of Korea (A070001).

\section{DISCLAIMER}

The funding sources had no role in the study design. The financial supporters neither had control over the collection, analysis or interpretation of any study data, nor did they control the writing of the article or the decision to submit the manuscript for publication.

1 Kearney PM, Whelton M, Reynolds K, Muntner P, Whelton PK, He J. Global burden of hypertension: analysis of worldwide data. Lancet 2005; 365: 217-223.

2 Kaplan NM. Systemic hypertension:mechanisms and diagnosis. In: Zipes DP, Libby P, Bonow RO and Braunwald E (eds). Braunwald's Heart Disease. Saunders Elsevier, Philadelphia, PA, USA, 2005, pp 959-987.

3 Cianci R, Citro F, Migneco A, Baldoni F, Minisci MC, Di Daniele N, De Lorenzo A Body fluid compartments in hypertension. Eur Rev Med Pharmacol Sci 2006; 10 75-78.

4 Raison J, Achimastos A, Bouthier J, London G, Safar M. Intravascular volume, extracellular fluid volume, and total body water in obese and nonobese hypertensive patients. Am J Cardiol 1983; 51: 165-170.

5 Raison J, Achimastos A, Asmar R, Simon A, Safar M. Extracellular and interstitial fluid volume in obesity with and without associated systemic hypertension. Am J Cardiol 1986; 57: 223-226

6 Tarazi RC. Hemodynamic role of extracellular fluid in hypertension. Circ Res 1976; 38 73-83.

7 Editorial: Volume-dependent essential hypertension? Br Med J 1974; 1: 470.

8 Harrap SB, Cumming AD, Davies DL, Foy CJ, Fraser R, Kamitani A, Connor JM, Lever AF, Watt GC. Glomerular hyperfiltration, high renin, and low-extracellular volume in high blood pressure. Hypertension 2000; 35: 952-957.

9 Bauer JH, Brooks CS. Body-fluid composition in normal and hypertensive man. Clin Sci (Lond) 1982; 62: 43-49.

10 Ishii M, Ohno K. Comparisons of body fluid volumes, plasma renin activity, hemodynamics and pressor responsiveness between juvenile and aged patients with essential hypertension. Jpn Circ J 1977; 41: 237-246.

11 Guyton AC, Hall JE. The body fluid compartments: Extracellular and intracellular fluids; interstitial fluid and edema. In: Guyton AC and Hall JE (eds). Textbook of Medical Physiology. Elsevier Saunders, Philadelphia, PA, USA, 2005, pp 291-306.

12 Kuhlmann MK, Zhu F, Seibert E, Bioimpedance Levin NW. dry weight and blood pressure control: New methods and consequences. Curr Opin Nephrol Hypertens 2005; 14: 543-549.

13 Valensise H, Andreoli A, Lello S, Magnani F, Romanini C, De Lorenzo A. Multifrequency bioelectrical impedance analysis in women with a normal and hypertensive pregnancy. Am J Clin Nutr 2000; 72: 780-783.

14 Toda Y, Segal N, Toda T, Morimoto T, Ogawa R. Lean body mass and body fat distribution in participants with chronic low back pain. Arch Intern Med 2000; 160 3265-3269.

15 Van Loan MD, Kopp LE, King JC, Wong WW, Mayclin PL. Fluid changes during pregnancy: use of bioimpedance spectroscopy. J Appl Physiol, 1995; 78: 1037-1042.

16 De Lorenzo A, Andreoli A, Matthie J, Withers P. Predicting body cell mass with bioimpedance by using theoretical methods: a technological review. J App/ Physiol 1997; 82: 1542-1558.

17 Kyle UG, Bosaeus I, De Lorenzo AD, Deurenberg P, Elia M, Gomez JM, Heitmann BL, Kent-Smith L, Melchior JC, Pirlich M, Scharfetter H, Schols AM, Pichard C. Bioelectrical impedance analysis-part i: review of principles and methods. Clin Nutr 2004; 23: 1226-1243.

18 Kyle UG, Bosaeus I, De Lorenzo AD, Deurenberg P, Elia M, Manuel Gomez J, Lilienthal Heitmann B, Kent-Smith L, Melchior JC, Pirlich M, Scharfetter H, MWJS A, Pichard C. Bioelectrical impedance analysis-part ii: Utilization in clinical practice. Clin Nutr 2004; 23: 1430-1453.

19 Armstrong LE. Hydration assessment techniques. Nutr Rev 2005; 63: S40-S54.

20 Ritz P. Body water spaces and cellular hydration during healthy aging. Ann N Y Acad Sci 2000; 904: 474-483.

21 Volgyi E, Tylavsky FA, Lyytikainen A, Suominen $H$, Alen M, Cheng S. Assessing body composition with dxa and bioimpedance: effects of obesity, physical activity, and age. Obesity (Silver Spring) 2008; 16: 700-705.

22 Ritz P, Vol S, Berrut G, Tack I, Arnaud MJ, Tichet J. Influence of gender and body composition on hydration and body water spaces. Clin Nutr 2008; 27: 740-746.

23 Strain GW, Wang J, Gagner M, Pomp A, Inabnet WB, Heymsfield SB. Bioimpedance for severe obesity: comparing research methods for total body water and resting energy expenditure. Obesity 2008; 16: 1953-1956.

24 Jaffrin MY, Morel H. Body fluid volumes measurements by impedance: a review of bioimpedance spectroscopy (bis) and bioimpedance analysis (bia) methods. Med Eng Phys 2008; 30: 1257-1269.

25 Lukaski HC Siders WA, Nielsen EJ, Hall CB. Total body water in pregnancy: Assessment by using bioelectrical impedance. Am J Clin Nutr 1994; 59: 578-585.

26 International Diabetes Institute, WHO Regional Office for the Western Pacific. The Asia-pacific Perspective: Redefining Obesity and its Treatment. Melbourne: Health Communications Australia Pty Limit, 2000.

27 Lukaski HC. Methods for the assessment of human body composition: traditional and new. Am J Clin Nutr 1987; 46: 537-556.

28 Kotsis V, Stabouli S, Papakatsika S, Rizos Z, Parati G. Mechanisms of obesity-induced hypertension. Hypertens Res 2010; 33: 386-393. 
29 Wang J, Pierson RN Jr. Disparate hydration of adipose and lean tissue require a new model for body water distribution in man. J Nutr 1976; 106: 1687-1693.

30 Wang Z, Deurenberg P, Wang W, Pietrobelli A, Baumgartner RN, Heymsfield SB. Hydration of fat-free body mass: new physiological modeling approach. Am J Physiol 1999; 276: E995-E1003.

31 Campbell DJ. Circulating and tissue angiotensin systems. J Clin Invest 1987; 79: 1-6.

32 Narkiewicz K. Diagnosis and management of hypertension in obesity. Obes Rev 2006; 7: $155-162$.
33 Marken Lichtenbelt WD, Fogelholm M. Increased extracellular water compartment, relative to intracellular water compartment, after weight reduction. J App/ Physiol 1999; 87: 294-298.

34 Engeli S, Bohnke J, Gorzelniak K, Janke J, Schling P, Bader M, Luft FC, Sharma AM. Weight loss and the renin-angiotensin-aldosterone system. Hypertension 2005; 45: 356-362.

35 Tarazi RC, Frohlich ED, Dustan HP.. Plasma volume in men with essential hypertension. N Engl J Med 1968; 278: 762-765. 